\title{
Photonic Band Gap from a Stack of Positive and Negative Index Materials
}

\author{
Jensen Li, Lei Zhou, C.T. Chan, ${ }^{*}$ and P. Sheng \\ Department of Physics, The Hong Kong University of Science and Technology, Clear Water Bay, Kowloon, Hong Kong, China
}

(Received 27 August 2002; published 25 February 2003)

\begin{abstract}
Layered heterostructures combining ordinary and negative refractive index materials are shown to display a new type of photonic band gap corresponding to zero (volume) averaged refractive index. Distinct from band gaps induced by Bragg scattering, the zero- $\bar{n}$ gap is invariant upon a change of scale length and is insensitive to disorder that is symmetric in the random variable. A metallic structure that exhibits such a band gap is explicitly designed, and its properties are calculated with accurate finite difference time domain simulations.
\end{abstract}

DOI: 10.1103/PhysRevLett.90.083901

Photonic band gap (PBG) material is a type of artificial composite with novel electromagnetic (EM) wave characteristics [1]. Conventional PBG materials have periodically modulated dielectric function and the photonic gap is a consequence of Bragg scattering [1]. As such, the PBG frequency is inversely proportional to the lattice constant, and randomness will destroy the band gap [2]. Recently demonstrated negative refractive index (negative- $n$ ) materials [3-5], which have simultaneously negative $\varepsilon$ and $\mu$, is another type of metamaterials with very special EM wave properties. In this Letter, we show that stacking alternating layers of ordinary (positive- $n$ ) and negative- $n$ materials can lead to a new type of photonic gap with properties very different from that of a Bragg gap. This new type of gap, denoted the zero- $\bar{n}$ gap, arises naturally when the volume averaged effective refractive index $(\bar{n})$ equals zero. Stop bands of this type can only be achieved in composites containing both positive- $n$ materials and negative- $n$ materials, but not in heterostructures containing one type alone (either positive or negative $n$ ).

We start by showing that in a one-dimensional (1D) layered stack, $\bar{n}=0$ implies the existence of a spectral gap. Numerical calculations are performed to demonstrate the unusual properties of this new type of photonic gap, assuming dispersions that are most frequently adopted for negative $\varepsilon$ and $\mu$. We demonstrate that the zero- $\bar{n}$ gap is invariant with respect to a (length) scale change, and insensitive to randomness as long as the $\bar{n}=$ 0 condition is satisfied. An explicit design of a metallic structure is given which exhibits such unconventional stop bands, and its properties are simulated by finitedifference-time-domain (FDTD) [6] solution of Maxwell equations, without assuming any effective $\varepsilon$ or $\mu$.

We first consider a 1D layered photonic crystal described by a periodic frequency-dependent permittivity $\varepsilon(x+a)=\varepsilon(x)$ and permeability $\mu(x+a)=\mu(x)$, where $a$ is the lattice constant. Its dispersion relation $\omega(\kappa)$ can be obtained by solving the equation for the $E$ field:

$$
-\frac{Z(x)}{n(x)} \frac{d}{d x}\left[\frac{1}{Z(x) n(x)} \frac{d E(x)}{d x}\right]=\left(\frac{\omega}{c}\right)^{2} E(x),
$$

PACS numbers: 42.70.Qs, 41.20.Jb, 73.20.Mf

where $n(x)=\sqrt{\varepsilon(x)} \sqrt{\mu(x)}$ and $Z(x)=\sqrt{\mu(x)} / \sqrt{\varepsilon(x)}$ are the refractive index and impedance at frequency $\omega$, respectively. Imposing the periodicity constraint $E(x+a)=e^{i \kappa a} E(x)$ leads to the solution condition.

$$
\operatorname{Tr}[T(\omega)]=2 \cos \kappa a,
$$

where $T(\omega)$ is a $2 \times 2$ transfer matrix [7]. For a doublelayer unit-cell ( $A B A B \ldots$ stack), we have

$$
\begin{aligned}
\operatorname{Tr}[T(\omega)]= & 2 \cos \left(\frac{\bar{n} \omega a}{c}\right)-\left(\frac{Z_{1}}{Z_{2}}+\frac{Z_{2}}{Z_{1}}-2\right) \\
& \times \sin \left(\frac{n_{1} \omega d_{1}}{c}\right) \sin \left(\frac{n_{2} \omega d_{2}}{c}\right),
\end{aligned}
$$

where $n_{i}, Z_{i}, d_{i}$ are, respectively, the refractive index, impedance, and thickness of the $i$ th layer. The first term corresponds to a homogeneous medium of average refractive index $\bar{n}=\frac{1}{a} \int_{0}^{a} n(x) d x$, and the second term is responsible for gap opening if there is an impedance mismatch. For the special case of matched impedance ( $Z_{1}=Z_{2}=Z_{0}$, a constant), the dispersion relationship is given by

$$
\kappa_{0} a=\bar{n} \omega a / c .
$$

In general $\left(Z_{1} \neq Z_{2}\right)$, when

$$
\kappa_{0} a=(\bar{n} \omega / c) a=m \pi, \quad(m \in \text { integers }) .
$$

Equation (3) becomes

$$
|\operatorname{Tr}[T(\omega)]|=\left|2+\left(\frac{Z_{1}}{Z_{2}}+\frac{Z_{2}}{Z_{1}}-2\right) \sin ^{2}\left(\frac{n_{1} \omega d_{1}}{c}\right)\right| \geq 2 .
$$

Except for $n_{1} d_{1} \omega / c$ being an integral multiple of $\pi$, Eq. (6) implies that Eq. (2) has no real solution for $\kappa$, indicating a spectral gap. This is the familiar Bragg condition. For conventional PBG materials, we can find multiple values of $\omega$ that satisfy the Bragg condition, leading to photonic band gaps at these frequencies. However, if we mix both positive and negative- $n$ materials to form a PBG material, there is an extra possibility that 


$$
\bar{n}=0,
$$

which also leads to Eq. (6) and thus a spectral gap. We emphasize that the $\bar{n}=0$ gap is not a Bragg gap at $\Gamma$ due to the folding of bands. The gap arising from $\bar{n}=0$ differs fundamentally from the usual Bragg gap, as we shall demonstrate. In particular, the frequency corresponding to the $\bar{n}=0$ gap is independent of the lattice constant, while all Bragg gap frequencies must scale with the lattice constant.

The $\bar{n}=0$ condition for the spectral gap is not limited to a periodic $\mathrm{AB}$ stacking, but is generally true in $1 \mathrm{D}$ periodic systems. Equation (1) is equivalent to two coupled first order differential equations:

$$
\begin{aligned}
\frac{d E_{+}(x)}{d x} e^{i(\omega / c) \int_{0}^{x} n d x^{\prime}}= & -\frac{d E_{-}(x)}{d x} e^{-i(\omega / c) \int_{0}^{x} n d x^{\prime}} \\
=\frac{1}{2 Z} \frac{d Z}{d x} & {\left[E_{+}(x) e^{i(\omega / c) \int_{0}^{x} n d x^{\prime}}\right.} \\
& \left.-E_{-}(x) e^{-i(\omega / c) \int_{0}^{x} n d x^{\prime}}\right],
\end{aligned}
$$

where the $E$ field is decomposed as forward and backward propagating components [8],

$$
E(x)=E_{+}(x) e^{i(\omega / c) \int_{0}^{x} n d x^{\prime}}+E_{-}(x) e^{-i(\omega / c) \int_{0}^{x} n d x^{\prime}} .
$$

For periodic systems, we seek Bloch solutions satisfying

$$
\begin{aligned}
E_{+}(a) e^{i \bar{n}(\omega / c) a} & =e^{i \kappa a} E_{+}(0), \\
E_{-}(a) e^{-i \bar{n}(\omega / c) a} & =e^{i \kappa a} E_{-}(0) .
\end{aligned}
$$

Let there be a certain frequency $\omega_{0}$ such that $\bar{n}\left(\omega_{0}\right)=$ 0 . If $Z(x)=Z_{0}$ (perfect impedance matching), Eq. (8) has two degenerate solutions at $\omega_{0}$, both with $\kappa=0$ and the general solution is given by their linear combination $E^{(0)}(x)=\alpha e^{i\left(\omega_{0} / c\right) \int_{0}^{x} n d x^{\prime}}+\beta e^{-i\left(\omega_{0} / c\right) \int_{0}^{x} n d x^{\prime}}$. When $Z(x)=Z_{0}+\Delta Z(x)$, with $\Delta Z(x)$ arbitrary but periodic, solving Eq. (8) [with Eq. (10)] by taking both $E(x)$ and $\kappa$ up to first order in $\Delta Z(x)$, it is straightforward to show that

$$
\kappa= \pm i \frac{1}{a}\left|\int_{0}^{a} \frac{1}{2 Z_{0}} \frac{d \Delta Z(x)}{d x} e^{2 i\left(\omega_{0} / c\right) \int_{0}^{x} n d x^{\prime}} d x\right| .
$$

Since $\kappa$ is imaginary, there must be a gap at $\omega_{0}$ if $\bar{n}\left(\omega_{0}\right)=0$.

To illustrate the basic ideas, we consider a simple 1D system with alternating nondispersive layers of $n>0$ and $n<0$ such that the structural and material parameters (given in the caption of Fig. 1) are chosen to satisfy $\bar{n}=0$. The dispersion relationship $\omega(\kappa)$ and the transmittance through a 32-unit slab as a function of frequency are shown in Fig. 1. The condition $\bar{n}=0$ is satisfied for all
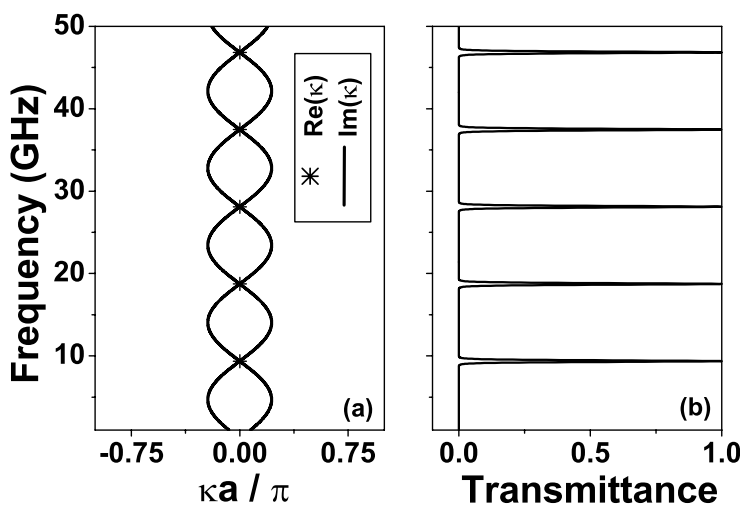

FIG. 1. (a) Dispersion relationship of a photonic crystal with unit cell consisting of one layer of air (thickness $=16 \mathrm{~mm}$ ) and one layer of negative- $n$ material $(\varepsilon=-8, \mu=-2$, thickness $=4 \mathrm{~mm}$ ); (b) Transmittance through a 1D photonic crystal slab consisting of 32 such unit cells.

frequencies in this model system. The numerical solution shows that the photonic gap covers all frequencies (no transmission and $\kappa$ purely imaginary) except for singular frequency points where $n_{1} d_{1} \omega / c$ are integral multiples of $\pi$, agreeing with Eq. (6). However, below we show these singular frequency points will disappear if $n$ is frequency dependent. For those frequencies satisfying $\bar{n}\left(\omega_{0}\right)=0$ and $n_{1} d_{1} \omega_{0} / c$ being an integral multiple of $\pi$, we have $\operatorname{Tr}\left[T\left(\omega_{0}\right)\right]=2$, and near $\omega_{0}$,

$$
\begin{aligned}
\operatorname{Tr}[T(\omega)] \approx & 2-\left(\left.Z_{1} \frac{d n_{1} \omega}{d \omega}\right|_{\omega_{0}} d_{1}+\left.Z_{2} \frac{d n_{2} \omega}{d \omega}\right|_{\omega_{0}} d_{2}\right) \\
& \times\left(\left.\frac{1}{Z_{1}} \frac{d n_{1} \omega}{d \omega}\right|_{\omega_{0}} d_{1}+\left.\frac{1}{Z_{2}} \frac{d n_{2} \omega}{d \omega}\right|_{\omega_{0}} d_{2}\right) \\
& \times\left(\frac{\omega-\omega_{0}}{c}\right)^{2},
\end{aligned}
$$

which is always less than two if we enforce the general condition on the dispersion, $\frac{d \varepsilon(\omega) \omega}{d \omega}>0$ and $\frac{d \mu(\omega) \omega}{d \omega}>0$; which ensures a positive definite energy density [3]. Equation (12) implies that we have a gap with zero width near $\omega_{0}$. Those singular isolated states in Fig. 1 corresponding to $n_{1} d_{1} \omega / c$ being an integral multiple of $\pi$ are thus artifacts of nondispersive media.

We note that all negative- $n$ materials that have been made [3-5], with simultaneously negative $\varepsilon$ and $\mu$, are dispersive. The negative refractive index originates from resonance, and $n(\omega)$ typically ranges from zero to a very negative value in the frequency range of negative refractive index [9]. When we stack positive- $n$ material and dispersive negative- $n$ material to form a layered structure, we can always find a $\omega$ within the range of negative refractive index such that $\bar{n}=0$. Thus, we do not have to do anything special to achieve the $\bar{n}=0$ condition for heterostructures comprising both positive and negative $n$ materials; such a condition will be met for some particular frequency. 
We give here numerical results for a $1 \mathrm{D}$ system with alternate layers of air ( $n=1$, as a positive- $n$ material) and dispersive negative- $n$ materials, with effective $\varepsilon(\omega)$ and $\mu(\omega)$ given by

$$
\begin{aligned}
& \varepsilon(f)=1+\frac{5^{2}}{0.9^{2}-f^{2}}+\frac{10^{2}}{11.5^{2}-f^{2}}, \\
& \mu(f)=1+\frac{3^{2}}{0.902^{2}-f^{2}},
\end{aligned}
$$

where $f$ is the frequency measured in GHz. Numerical values of $\varepsilon(\omega)$ and $\mu(\omega)$ are given in Fig. 2(a). The band structure is shown in Fig. 2(b), while the solid lines in Fig. 2(c) give the transmittance through a stack of 16 unit cells. The band structure and the transmittance clearly show two band gaps. The $\bar{n}$ of the system (air plus the negative- $n$ material) is zero at $2.3 \mathrm{GHz}$, and a gap does open at that frequency. We see another gap at about $8 \mathrm{GHz}$, which originates from Bragg scattering.

The gap due to $\bar{n}=0$ possesses some unique properties that distinguish itself from a Bragg gap. A Bragg gap is an intrinsic consequence of periodicity, and the gap frequency is tied with the size of the unit-cell. When we change the unit-cell size by a scaling factor, a Bragg gap will shift in frequency accordingly. However, the $\bar{n}=0$ gap is independent of periodicity and remains invariant with scaling. This is demonstrated in Fig. 2(c). The solid line in Fig. 2(c) is the transmission that corresponds to the band structure in Fig. 2(b); while the dotted line is the transmittance through the same material, but the lattice constant $a$ is scaled by a factor of $2 / 3$. The Bragg gap that was originally near eight $\mathrm{GHz}$ shifts upwards in frequency, as expected. However, the $\bar{n}=0$ gap remains unchanged at $2.3 \mathrm{GHz}$.

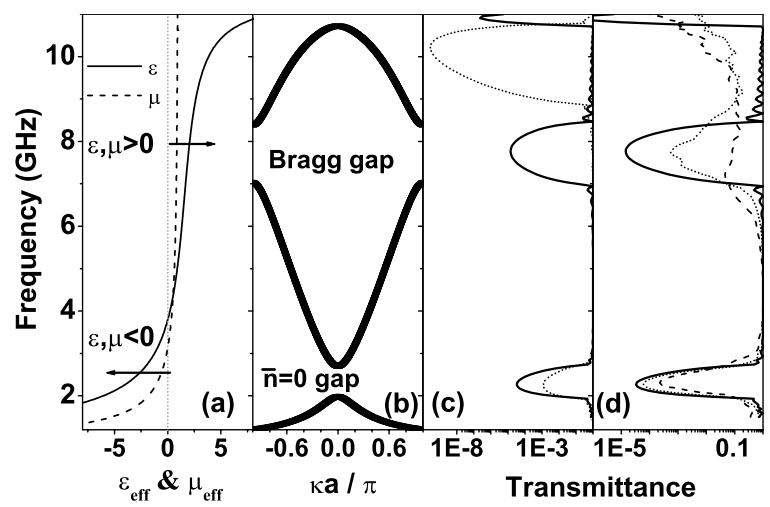

FIG. 2. (a) Effective $\varepsilon$ and $\mu$ of the negative- $n$ material, as given by Eq. (13); (b) Dispersion relationship of a photonic crystal with alternate layers of air (12 mm thick) the negative- $n$ material $(6.0 \mathrm{~mm}$ thick) with material parameter as shown in (a); (c) Solid line: Transmittance through 16 unit cells, corresponding to the band structure in (b). Dotted line: Transmittance when the lattice constant is scaled by 2/3; (d) Transmittance through 16 unit cells, with various degree of disorder in thickness. See text for details.
The Bragg gap is sensitive to deviation from periodic order [2]. Figure 2(d) compares the transmittance through a 16 unit-cell stack with structural and material parameters that give the band structure in Fig. 2(b) for different degrees of disorder. The solid line corresponds to a perfectly ordered stack, while the dotted line and dashed line corresponds to the transmission through stacks with thickness deviation (random uniform deviate) of \pm 3 and $\pm 6 \mathrm{~mm}$, respectively, each ensemble averaged over 24 realizations. As expected, the Bragg gap is destroyed by strong disorder, but the $\bar{n}=0$ gap survives. The robustness of the $\bar{n}=0$ gap comes from the fact that the $\bar{n}(\omega)=0$ solution remains invariant under disorder that is symmetric ( + and - equally probable).

With the help of accurate FDTD simulations [10], we have designed a structure that exhibits such an unconventional stop band. A building block for the negative- $n$ material is shown in Fig. 3. The rectangular metallic split ring resonators on the left and right gives negative $\mu$, while the metal fork in the middle gives negative $\varepsilon$. This structure is designed so as to (i) ensure that effective $\mu$ and $\varepsilon$ (and hence effective $n$ ) can be meaningfully defined near $\bar{n}(\omega)=0$, and (ii) optimize the impedance matching in the pass bands with vacuum. All metallic lines have thickness of $0.2 \mathrm{~mm}$. A $1.6 \mathrm{~mm}$ dielectric spacer layer $\left(\varepsilon_{r}=5.3\right)$ separates the resonator rings and the fork. The basic building block is replicated to tile the $\vec{E}-\vec{H}$ plane (see Fig. 3 for the direction of $\vec{E}$ and $\vec{H}$ ) with lattice constants of $16 \mathrm{~mm}$ in the $\vec{E}$ direction and $6 \mathrm{~mm}$ in the $\vec{H}$ direction. The resulting structure is effectively a $3.5-$ mm-thick negative- $n$ slab placed on the $\vec{E}-\vec{H}$ plane. We first calculate the transmission and reflection spectra through such a single negative- $n$ slab by FDTD simulation, and then derive its effective $\varepsilon(\omega)$ and $\mu(\omega)$ from the calculated spectra (both amplitude and phase) [11], treating the slab as an effective medium. The effective $\varepsilon(\omega)$ and $\mu(\omega)$ are plotted in Fig. 4(a) [12]. Between 4.1 and $4.8 \mathrm{GHz}$, both $\varepsilon_{\text {eff }}$ and $\mu_{\text {eff }}$ are found to be negative due to resonance, demonstrating that the material has an effectively negative refractive index in this frequency regime.

This negative- $n$ slab is then repeated in the $\vec{k}$ direction, with a $7 \mathrm{~mm}$ air space (serving as the positive- $n$ medium)

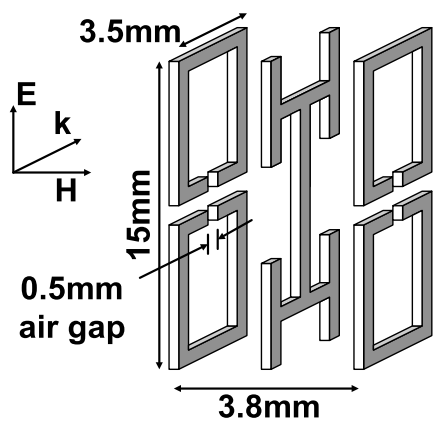

FIG. 3. Structural details of the negative- $n$ material. 


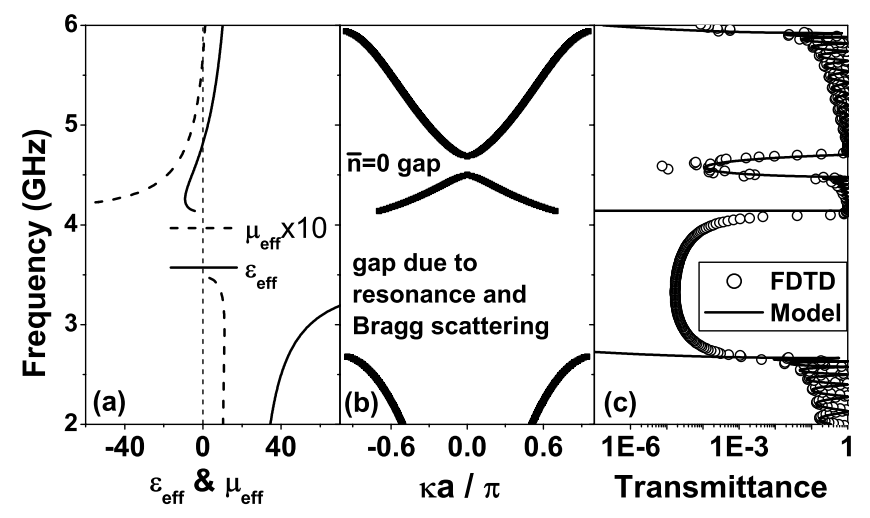

FIG. 4. (a) $\varepsilon_{\text {eff }}$ and $\mu_{\text {eff }}$ as functions of frequency [12] of the negative- $n$ material shown in Fig. 3; (b) band structure for a photonic crystal with alternating layers of air ( $7 \mathrm{~mm}$ thick) and the designed negative- $n$ material [thickness $=3.5 \mathrm{~mm}$, $\varepsilon_{\text {eff }}, \mu_{\text {eff }}$ shown in (a)]; (c) Transmittance through a slab consisting of 16 unit cells with details described above, through direct FDTD simulation (open circles) and material properties represented by $\varepsilon_{\text {eff }}$ and $\mu_{\text {eff }}$ (solid line) [12].

in between. This creates a 1D photonic crystal with unitcell consisting of a negative- $n$ slab and a positive- $n$ one. We have calculated the transmission spectra for a 16-unitcell slab, using FDTD simulations. The result is shown as open circles in Fig. 4(c). The FDTD result represents a "brute-force" solution of the Maxwell equations, with the microstructure shown in Fig. 3 taken fully into account. The only approximation is the perfect metal boundary conditions between metal-dielectric interfaces.

We have also calculated the band structure [Fig. 4(b)] of 1D photonic crystal comprising alternating layers of designed negative- $n$ material and air, with the predetermined $\varepsilon_{\text {eff }}(\omega)$ and $\mu_{\text {eff }}(\omega)$ of the negative- $n$ slab. The transmittance through a 16-unit-cell slab is shown as the solid line in Fig. 4(c). Both the band structure [Fig. 4(b)] and the transmission spectra clearly show two band gaps. We note in Fig. 4(c) the agreement between the rigorous FDTD (open circles) result and those calculated using the $\varepsilon_{\text {eff }}(\omega)$ and $\mu_{\text {eff }}(\omega)$ (solid line). It shows that the negative- $n$ slab can indeed be treated as an effective medium, except between $2.7 \sim 4.1 \mathrm{GHz}$, where effective medium theory fails either because the scattering within or between the slabs are strong [12]. We also calculated $\bar{n}(\omega)$ for the combined system, and found that $\bar{n}=0$ at about $4.5 \mathrm{GHz}$, which corresponds to the gap at 4.5 GHz. We emphasize that nearly identical results are obtained with FDTD. This again reinforces the notion that $\bar{n}=0$ does give rise to gaps, and we have an explicit structure which can demonstrate this effect.

To summarize, we showed that $\bar{n}=0$ implies photonic band gaps. While both negative- $n$ and positive- $n$ materials are transparent, these transparent slabs can become nearly totally reflecting when they are stacked together for a certain range of wavelengths near $\bar{n}(\omega)=0$ that can be much longer than the repeat distance of the heterostructure. These results are rather intriguing, because for positive- $n$ materials, layered structures are always transparent if each layer is both transparent and thin compared to the wavelength, as dictated by the effective medium limit.

This work is supported by Hong Kong RGC through HKUST6138/00P.

*Email address: phchan@ust.hk

[1] E. Yablonovitch, Phys. Rev. Lett. 58, 2059 (1987); S. John, Phys. Rev. Lett. 58, 2486 (1987).

[2] Z. Y. Li and Z. Q. Zhang, Phys. Rev. B 62, 1516 (2000); M. M. Sigalas et al., Phys. Rev. B 59, 12767 (1999); A. A. Asatryan et al., Phys. Rev. E 62, 5711 (2000); S. Fan et al., J. Appl. Phys. 78, 1415 (1995).

[3] V. G. Veselago, Sov. Phys. Usp. 10, 509 (1968).

[4] J. B. Pendry et al., IEEE Trans. Microwave Theory Tech. 47, 2075 (1999); J. B. Pendry, Phys. Rev. Lett. 85, 3966 (2000).

[5] D. R. Smith et al., Phys. Rev. Lett. 84, 4184 (2000); R. A. Shelby et al., Appl. Phys. Lett. 78, 489 (2001); R. A. Shelby, D. R. Smith, and S. Schultz, Science 292, 77 (2001).

[6] K. S. Yee, IEEE Trans. Antennas Propag. 14, 302 (1966).

[7] See, e.g., K. Busch, C.T. Chan, and C. M. Soukoulis, in Photonic Band Gap Materials, edited by C. M. Soukoulis (Kluwer, Dordrecht, 1996).

[8] Forward and backward here are defined by the group velocity.

[9] See, e.g., Eq. (15) in D. R. Smith and N. Kroll, Phys. Rev. Lett. 85, 2933 (2000).

[10] Simulations are performed using CONCERTO 2.0, Vector Fields Limited, England, 2001.

[11] See, e.g., D. R. Smith et al., Phys. Rev. B 65, 195104 (2002).

[12] Between 3.4 and $4.1 \mathrm{GHz}$, the $\varepsilon_{\text {eff }}$ and $\mu_{\text {eff }}$ are found to be complex, which is unphysical since there is no absorption in the FDTD simulation. This indicates that multiple scattering effect is strong and an effective medium description is inadequate in this range. Between 2.7 and $3.4 \mathrm{GHz}$, the large $\varepsilon_{\text {eff }}$ is due to resonance. Coupling between adjacent negative- $n$ slabs are strong near resonance, making the use of effective refractive indices of a single plate inaccurate for modeling the composite system. The solid line in Fig. $4(\mathrm{c})$ is obtained using $\varepsilon_{\text {eff }}$ and $\mu_{\text {eff }}$ "as is," and the agreement is expected to be bad from 2.7 to $4.1 \mathrm{GHz}$ where effective medium fails. Agreement is very good in other frequencies. 